\title{
ÍNDICES DE CONFORMAÇÃO CORPORAL DE EQUÍDEOS CRIADOS NO PANTANAL
}

(Body conformation indices of horses created in pantanal)

Marcos Paulo Gonçalves Rezende ${ }^{1 *}$, Julio Cesar Souza ${ }^{2}$, Nicacia Monteiro Oliveira ${ }^{2}$

1Universidade Estadual do Sudoeste da Bahia. *Corresponding author: mpgrezende@gmail.com
2Universidade Federal Do Mato Grosso Do Sul

RESUMO: Os índices de conformação corporal (ICC) possibilitam estimar aptidões de equinos. Não existem estudos que avaliem ICC em diferentes grupos genéticos (GG) de equídeos no Pantanal. Objetivou-se avaliar ICC de 452 equídeos distribuídos em Árabe, Quarto de Milha, Pantaneiro, Crioulo, equinos e muares mestiços. Mensuram-se medidas lineares e estimaram-se ICC. Para análise da diversidade entre GG, utilizaram-se procedimentos univariados e multivariados. Os ICC apresentam diferenças entre os GG criados no Pantanal. Hipotetiza-se que os produtores têm optado também por utilizar GG exóticos na produção de equinos e muares mestiços, visto pela distância euclidiana entre esses GG. Equinos e muares mestiços fêmeas, Pantaneiros, possuem menores aptidões para tarefas que exigem força, menor porte corporal e capacidade de carga, aptidões para sela em relação GG exóticos. Apesar do Pantaneiro apresentar menor aptidão para atividades que exijam força e capacidade de carga em relação às raças exóticas, não houve diferença de classificação nos ICC suficiente para mudar o tipo funcional dos GG, e considerando que esta raça possui perfil eumétrico e adaptada ao Pantanal, os produtores podem optar seu uso quando se busca agregar também a rusticidade ao seu rebanho.

Palavra-chave: Equus caballus; Equus asinus; genótipos; índice de conformação; região Pantaneira

ABSTRACT: The body conformation indexes (ICC) allows the estimation of equine aptitudes. There are no studies evaluating ICC in different genetic groups (GG) of equidae in the Pantanal.The objective of this study was to evaluate the ICC of 452 equidae distributed in Arabian, Quarter Horse, Pantaneiro, Crioulo, mestizo horses and mules. Linear measurements were measured and ICC was estimated. For analysis of the diversity between GG, univariate and multivariate procedures were used. The ICC presents differences between the GG created in the Pantanal. It is hypothesized that producers have also opted to use exotic GG in the production of mestizo horses and mules, because of the Euclidean distance between these GG. Female mestizo horses and mules, Pantaneiros, have lower skills for tasks that require strength, lower body size, carrying capacity and saddle skills in relation to exotic GG. Although the Pantaneiro presented less fitness for activities that require strength and load capacity in relation to the exotic racea. There was no difference in classification in the ICC sufficient to change the functional type of GG and considering that this breed has a eumetric profile and adapted to the Pantanal, the producers can choose their use when seeking to add also the rusticity to their herd.

Keywords: Equus caballus; Equus asinus; Genotypes; Conformation index; Pantaneira region 


\section{INTRODUÇÃO}

No Pantanal, os equídeos são imprescindíveis para os trabalhos de manejo dos bovinos e para 0 deslocamento, percorrendo diariamente quilômetros entre as invernadas. Assim, definir o genótipo com modelo corporal mais adequado para esta rotina de atividades pode ser fundamental na diminuição do desgaste físico do animal (Nicolleti et al., 2000), além de permitir que seja expresso todo o seu potencial genético. Extrair informações sobre a diversidade fenotípica utilizando marcadores biométricos contribuem na distinguinção do grau de divergência das aptidões específicas de diferentes grupos genéticos (Rezende et al. 2016; Teixeira Neto et al. 2015; Souza et al., 2015), tornando-se também, um indicador de diferenças seletivas adaptativas entre populações (Biagiotti et al. 2013).

Nesse tocante, a avaliação de índices de conformação corporal pode ser uma ferramenta indicativa para verificar as aptidões dos equídeos, além de possuírem facilidade de estimação, por serem analisadas combinando medidas lineares mensuradas em diferentes regiões no corpo dos animais (Oom e Ferreira, 1987), além disso, a avaliação fenotípica descreve um indivíduo melhor que os métodos convencionais de pesagem e classificação (Pundir et al. 2011). Estes índices de conformação corporal isoladamente não bastam para 0 julgamento das habilidades de um equino, entretanto, constituem elementos auxiliares valiosos, principalmente em trabalhos de seleção (Torres e Jardim, 1992).

Através dos índices de conformoção corporal pode-se estimar o tipo funcional do animal como: aptdões para atividades equestres (brevilíneos, mediolíneos, longelíneos), estrutura corporal (hipermétrico, hipométrico e elipométrico), equilibrio e conformação em geral do corpo (Rezende et al., 2015; Torres e Jardim, 1981). Estudos com esta ferramenta vêm sendo realizados por vários autores (Franci et al., 1989; McManus et al., 2005; Rezende et al., 2018). Todavia, não existe um tipo de estudo com esta abordagem comparando os diferentes genótipos que compõem o rebanho de equídeos no Pantanal. Os resultados podem complementar os critérios de escolha de animais que serão criados e utilizados em atividades na região. Assim, o objetivo deste trabalho foi avaliar índices de conformação corporal entre diferentes grupos genéticos de equídeos criados no Pantanal do MS.

\section{MATERIAL E MÉTODOS}

Conduziu-se a pesquisa em parcerias com diferentes propriedades rurais que utilizam os equídeos no manejo de bovinos de corte na região do Pantanal de Aquidauana, Abobral, Rio Negro, Nhecolândia e Miranda (Brasil). Mensuraram-se 452 equídeos machos $(M)$ e fêmeas $(F)$ adultos, distribuídos nas seguintes raças: 28 animais Árabe $(\mathrm{M}=11 / \mathrm{F}=17), 97$ Quarto de Milha $(\mathrm{M}=24 / \mathrm{F}=73), 52$ Pantaneiro $(\mathrm{M}=29 / \mathrm{F}=23), 31$ Crioulo $(\mathrm{M}=18 / \mathrm{F}=13)$, 170 equinos mestiços $(M=93 / F=77)$ e 74 muares mestiços ( $M=32 / F=42)$.

Para cálculo dos índices de conformação corporal, utilizaram-se as combinações de medidas lineares, mensuradas com auxílio de fita métrica e hipômetro. As medidas foram de altura de cernelha $(A C)$, comprimento corporal $(\mathrm{CC})$, altura de garupa $(A G)$, perímetro de canela $(P C)$, perímetro de joelho (PJ), perímetro de antebraço (PAB), perímetro torácico $(\mathrm{PT})$, distância codilho ao solo e largura de peito (LP) (Oom e Ferreia, 1987; Torres e Jardim, 1992). Com base nas combinações dos resultados das medidas lineares $\mathrm{e}$ conforme Oom e Ferreira (1987) e Torres e Jardim (1992), estimaram-se 
os índices: relação cernelha e garupa (AC/AG), dáctilo torácico [(PC/PT)x100], peso corporal estimado em quilo $\left(\mathrm{PT}^{3} \mathrm{x} 80\right)$, índice corporal (CC/PT), conformação ( $\left.\mathrm{PT}^{2} / \mathrm{AC}\right)$, de carga 1 $\left[\left(\mathrm{PT}^{2} \times 56\right) / \mathrm{AC}\right]$ e carga $2\left[\left(\mathrm{PT}^{2} \times 95\right) / \mathrm{AC}\right]$, corporal relativo $[(C C \times 100) / A C]$, de compacidade [(Peso corporal estimado/AC)/100] e índice meloscópico $[D C S /(P A B+P C+P J)]$.

Os grupos genéticos foram separados por sexo, sendo portanto gerado um total de 12 "grupos genéticos". Para detectar possíveis diferenças entre os grupos genéticos, realizou-se a análise de ANOVA (SAS, 2000 ), seguido do teste de Tukey a $5 \%$ de significância. Objetivando detectar possíveis diferenças entre os grupos genéticos considerando simultaneamente os índices de conformação, realizou-se análise de MANOVA seguido do teste de Hotelling's no programa Past (Hammer et al., 2001). Para verificar as variáveis com maiores contribuições para diferenciação dos equídeos, utilizou-se análise de componentes principais.

Adicionalmente com objetivo de verificar diversidade entre os equídeos e sua relação com as variáveis mensuradas, elaborou-se no programa $\mathrm{R}$, um gráfico heatmap ( $\mathrm{R}$ Development Core Team, 2008). Para a elaboração do dendrograma (Cluster) agregado ao heatmap, foi considerado análise da distância euclidiana média com agrupamento por meio de ligação completa, sendo liberada a aproximação imparcial (Al) calculada através do rearranjo da multiescala dos bootstrap. Foi considerado que o cluster com valores acima de $95 \%$ de Al são fortemente suportados pelos dados.

\section{RESULTADOS E DISCUSSÃO}

Os índices de conformação corporal apresentaram variações $(P<0,05)$ entre os grupos genéticos, exceto para o índice de relação cernelha e garupa (Tabela 1). Para este índice, todos os grupos apresentaram valores variando de 1,00 a 1,03 . Quanto mais próximo de 1 for o valor desse índice maior será o equilíbrio entre os membros locomotores. Quando há um grande desequilíbrio entre alturas de cernelha e garupa, pode-se considerar efeito consequente da abertura anormal dos ângulos articulares dos membros anteriores e posteriores, podendo prejudicar tanto $\mathrm{o}$ andar como a resistência do equino (McManus et al., 2005).

Tabela 1. Valores médios e desvio padrão dos Índices de conformação dos equídeos puros e mestiços criados no Pantanal/MS.

\begin{tabular}{|c|c|c|c|c|c|c|c|c|}
\hline & \multicolumn{2}{|l|}{ PC } & \multicolumn{2}{|c|}{ IRCG } & \multicolumn{2}{|l|}{ IDT } & IC & IM \\
\hline & \multirow{2}{*}{\multicolumn{2}{|c|}{$\begin{array}{l}418,11 \pm 51, \\
99 \mathrm{C}\end{array}$}} & \multirow{2}{*}{\multicolumn{2}{|c|}{$\begin{array}{l}1,00 \pm 0, \\
02 \mathrm{~A}\end{array}$}} & \multirow{2}{*}{\multicolumn{2}{|c|}{$\begin{array}{l}0,11 \pm 0 \\
01 \mathrm{AB}\end{array}$}} & 0,83 & $0,91 \pm$ \\
\hline & & & & & & & $\pm 0,0$ & 0,05 \\
\hline \multirow{3}{*}{ AR_M } & \multirow{3}{*}{\multicolumn{2}{|c|}{$\begin{array}{l}516,79 \pm 84 \\
46 \mathrm{AB}\end{array}$}} & \multirow{3}{*}{\multicolumn{2}{|c|}{$\begin{array}{l}1,01 \pm 0, \\
01 \mathrm{~A}\end{array}$}} & & & & \\
\hline & & & & & \multirow{2}{*}{\multicolumn{2}{|c|}{$\begin{array}{l}0,10 \pm 0 \\
01 \mathrm{C}\end{array}$}} & 0,80 & $0,90 \pm$ \\
\hline & & & & & & & $\pm 0,0$ & 0,05 \\
\hline \multirow{2}{*}{ AR_F } & \multirow{3}{*}{\multicolumn{2}{|c|}{$\begin{array}{l}450,05 \pm 45, \\
50 B\end{array}$}} & \multirow{3}{*}{\multicolumn{2}{|c|}{$\begin{array}{l}1,01 \pm 0, \\
02 \mathrm{~A}\end{array}$}} & \multirow{2}{*}{\multicolumn{2}{|c|}{$\begin{array}{l}0,12 \pm 0, \\
01 \mathrm{AB}\end{array}$}} & 083 & AD \\
\hline & & & & & & & $\begin{array}{r}0,03 \\
+0,0\end{array}$ & 0.07 \\
\hline \multirow[t]{2}{*}{ CR_M } & & & & & & & $2 A$ & B \\
\hline & \multirow{2}{*}{\multicolumn{2}{|c|}{$\begin{array}{l}416,90 \pm 36 \\
88 C\end{array}$}} & \multicolumn{2}{|c|}{$\begin{array}{l}1,01 \pm 0, \\
02 \mathrm{~A}\end{array}$} & \multicolumn{2}{|c|}{$\begin{array}{l}0,11 \pm 0, \\
01 \mathrm{AB}\end{array}$} & $\begin{array}{l}0,83 \\
\pm 0,0\end{array}$ & $\begin{array}{l}0,78 \pm \\
0,06\end{array}$ \\
\hline CR_F & & & & & & & $2 \mathrm{~A}$ & B \\
\hline $\begin{array}{l}\mathrm{MU}_{-} \\
\mathrm{M}\end{array}$ & \multicolumn{2}{|c|}{$\begin{array}{l}413,19 \pm 66 \\
88 C\end{array}$} & \multicolumn{2}{|c|}{$\begin{array}{l}1,01 \pm 0, \\
02 \mathrm{~A}\end{array}$} & \multicolumn{2}{|c|}{$\begin{array}{l}0,12 \pm 0 \\
01 \mathrm{~A}\end{array}$} & $\begin{array}{l}0,82 \\
\pm 0,0 \\
5 A\end{array}$ & $\begin{array}{l}0,85 \pm \\
0,05 \\
B\end{array}$ \\
\hline & \multicolumn{2}{|c|}{$\begin{array}{l}404,02 \pm 70 \\
58 \mathrm{C}\end{array}$} & \multicolumn{2}{|c|}{$\begin{array}{l}1,00 \pm 0, \\
03 \mathrm{~A}\end{array}$} & \multicolumn{2}{|c|}{$\begin{array}{l}0,12 \pm 0 \\
02 \mathrm{AB}\end{array}$} & $\begin{array}{l}0,83 \\
\pm 0,0 \\
8 A\end{array}$ & $\begin{array}{l}0,90 \pm \\
0,07 \\
A B\end{array}$ \\
\hline MU_F & \multirow{2}{*}{\multicolumn{2}{|c|}{$\begin{array}{l}397,88 \pm 55, \\
64 C\end{array}$}} & \multirow{2}{*}{\multicolumn{2}{|c|}{$\begin{array}{l}1,01 \pm 0, \\
02 \mathrm{~A}\end{array}$}} & \multirow{2}{*}{\multicolumn{2}{|c|}{$\begin{array}{l}0,11 \pm 0 \\
01 \mathrm{AB}\end{array}$}} & $\begin{array}{l}0,84 \\
\pm 0,0\end{array}$ & $\begin{array}{l}0,87 \pm \\
0,05\end{array}$ \\
\hline \multirow[t]{2}{*}{ PA_M } & & & & & & & $3 A$ & B \\
\hline & \multirow{2}{*}{\multicolumn{2}{|c|}{$\begin{array}{l}409,00 \pm 55 \\
77 C\end{array}$}} & \multirow{2}{*}{\multicolumn{2}{|c|}{$\begin{array}{l}1,00 \pm 0, \\
01 \mathrm{~A}\end{array}$}} & $\begin{array}{l}0,11 \\
01 \mathrm{~B}\end{array}$ & & $\begin{array}{l}0,85 \\
\pm 0,0\end{array}$ & $\begin{array}{l}0,92 \pm \\
0,06\end{array}$ \\
\hline PA_F & & & & & & & $3 A$ & A \\
\hline $\begin{array}{l}\text { QM_ } \\
M\end{array}$ & $\begin{array}{l}453,1 \\
53 B\end{array}$ & \pm 43 & $\begin{array}{l}1,0 \\
02 A\end{array}$ & & $\begin{array}{l}0,12 \\
01 \mathrm{AE}\end{array}$ & & $\begin{array}{l}0,84 \\
\pm 0,0 \\
3 A\end{array}$ & $\begin{array}{l}0,86 \pm \\
0,06 \\
\text { B }\end{array}$ \\
\hline & $\begin{array}{l}570,6 \\
6,26 A\end{array}$ & \pm 15 & $\begin{array}{l}1,0 \\
03 A\end{array}$ & & $\begin{array}{l}0,12 \\
01 \mathrm{AE}\end{array}$ & & $\begin{array}{l}0,75 \\
\pm 0,0\end{array}$ & $\begin{array}{l}0,91 \pm \\
0,08\end{array}$ \\
\hline QM_F & & & & & & & $9 \mathrm{~B}$ & $A B$ \\
\hline $\begin{array}{l}\mathrm{SRD}_{-} \\
\mathrm{M}\end{array}$ & $\begin{array}{l}429,8 \\
04 C\end{array}$ & \pm 63 & $\begin{array}{l}1,0 \\
02 A\end{array}$ & & $\begin{array}{l}0,11 \\
01 \mathrm{AE}\end{array}$ & & $\begin{array}{l}0,84 \\
\pm 0,0 \\
3 A\end{array}$ & $\begin{array}{l}0,86 \pm \\
0,06 \\
B\end{array}$ \\
\hline $\begin{array}{l}\mathrm{SRD}_{-} \\
\mathrm{F}\end{array}$ & $\begin{array}{l}412,9 \\
45 C\end{array}$ & \pm 72 & & \pm 0 & $\begin{array}{l}0,11 \\
01 C\end{array}$ & & $\begin{array}{l}0,84 \\
\pm 0,0 \\
5 A\end{array}$ & $\begin{array}{l}0,87 \pm \\
0,06 \\
B\end{array}$ \\
\hline & $F$ & ICG1 & & ICC & & ICF & & ICO \\
\hline & $04 \pm 0$ & 114,3 & & & $5 \pm$ & & $2 \pm 4,8$ & $2,84 \pm$ \\
\hline $\begin{array}{r}\text { AR } \\
\text { M }\end{array}$ & B & $6,71 \mathrm{~B}$ & & & & $O A$ & & $\begin{array}{l}0,25 \\
B\end{array}$ \\
\hline $\begin{array}{c}\text { AR } \\
F\end{array}$ & $\begin{array}{l}0 \pm 0 \\
A B\end{array}$ & $\begin{array}{l}128, \\
12,5\end{array}$ & & & $\begin{array}{l}26 \pm \\
A B B\end{array}$ & $7 \mathrm{~A}$ & $\pm 3,2$ & $\begin{array}{l}3,43 \pm \\
0,51 \\
\mathrm{AB}\end{array}$ \\
\hline
\end{tabular}




\begin{tabular}{|c|c|c|c|c|c|}
\hline $\begin{array}{r}\text { CR } \\
M\end{array}$ & $\begin{array}{l}2,18 \pm 0, \\
15 \mathrm{~B}\end{array}$ & $\begin{array}{l}121,99 \pm \\
8,18 \mathrm{~B}\end{array}$ & $\begin{array}{l}206,94 \pm \\
13,88 \mathrm{~B}\end{array}$ & $\begin{array}{l}101,06 \pm 3 \\
45 A\end{array}$ & $\begin{array}{l}3,10 \pm \\
0,31 \\
\text { B }\end{array}$ \\
\hline $\begin{array}{c}\mathrm{CR} \\
\mathrm{F}\end{array}$ & $\begin{array}{l}2,14 \pm 0, \\
11 \mathrm{~B}\end{array}$ & $\begin{array}{l}119,76 \pm \\
6,12 B\end{array}$ & $\begin{array}{l}203,17 \pm \\
10,38 \mathrm{~B}\end{array}$ & $\begin{array}{l}102,24 \pm 3 \\
56 \mathrm{~A}\end{array}$ & $\begin{array}{l}2,97 \pm \\
0,22 \\
B\end{array}$ \\
\hline $\begin{array}{l}\mathrm{MU} \\
\mathrm{M}\end{array}$ & $\begin{array}{l}2,14 \pm 0, \\
19 \mathrm{~B}\end{array}$ & $\begin{array}{l}120,02 \pm \\
10,47 \mathrm{~B}\end{array}$ & $\begin{array}{l}203,61 \pm \\
17,76 \mathrm{~B}\end{array}$ & $\begin{array}{l}101,45 \pm 3 \\
47 A\end{array}$ & $\begin{array}{l}2,97 \pm \\
0,41 \\
\text { B }\end{array}$ \\
\hline$\underset{\mathrm{F}}{\mathrm{MU}}$ & $\begin{array}{l}2,10 \pm 0, \\
21 \mathrm{~B}\end{array}$ & $\begin{array}{l}117,52 \pm \\
11,69 \mathrm{~B}\end{array}$ & $\begin{array}{l}199,37 \pm \\
19,83 B\end{array}$ & $\begin{array}{l}101,02 \pm 5 \\
62 A\end{array}$ & $\begin{array}{l}2,89 \pm \\
0,43 \\
\text { B }\end{array}$ \\
\hline $\begin{array}{l}\text { PA } \\
\text { M }\end{array}$ & $\begin{array}{l}2,04 \pm 0, \\
13 \mathrm{~B}\end{array}$ & $\begin{array}{l}114,10 \pm \\
7,18 \mathrm{~B}\end{array}$ & $\begin{array}{l}193,56 \pm \\
12,18 B\end{array}$ & $\begin{array}{l}100,65 \pm 3 \\
59 A\end{array}$ & $\begin{array}{l}2,78 \pm \\
0,30 \\
\text { B }\end{array}$ \\
\hline $\begin{array}{c}\text { PA } \\
\text { F }\end{array}$ & $\begin{array}{l}2,09 \pm 0, \\
17 \mathrm{~B}\end{array}$ & $\begin{array}{l}116,96 \pm \\
9,38 \mathrm{~B}\end{array}$ & $\begin{array}{l}198,41 \pm \\
15,91 B\end{array}$ & $\begin{array}{l}102,62 \pm 2 \\
37 A\end{array}$ & $\begin{array}{l}2,88 \pm \\
0,36 \\
\text { B }\end{array}$ \\
\hline $\begin{array}{l}\bar{Q} \\
M \\
M\end{array}$ & $\begin{array}{l}2,12 \pm 0, \\
12 B\end{array}$ & $\begin{array}{l}118,56 \pm \\
6,76 \mathrm{~B}\end{array}$ & $\begin{array}{l}201,13 \pm \\
11,46 B\end{array}$ & $\begin{array}{l}99,29 \pm 4,2 \\
4 A B\end{array}$ & $\begin{array}{l}3,02 \pm \\
0,26 \\
B\end{array}$ \\
\hline $\begin{array}{l}\mathrm{Q} \\
\mathrm{M}_{-} \\
\mathrm{F}^{-}\end{array}$ & $\begin{array}{l}2,46 \pm 0 \\
38 \mathrm{~A}\end{array}$ & $\begin{array}{l}137,68 \pm \\
21,36 A\end{array}$ & $\begin{array}{l}233,56 \pm \\
36,23 A\end{array}$ & $\begin{array}{l}95,99 \pm 8,1 \\
6 B\end{array}$ & $\begin{array}{l}3,81 \pm \\
0,94 \\
\text { A }\end{array}$ \\
\hline $\begin{array}{l}\text { SR } \\
D_{-} \\
M\end{array}$ & $\begin{array}{l}2,11 \pm 0, \\
17 \mathrm{~B}\end{array}$ & $\begin{array}{l}118,12 \pm \\
9,35 \mathrm{~B}\end{array}$ & $\begin{array}{l}200,38 \pm \\
15,86 B\end{array}$ & $\begin{array}{l}101,42 \pm 4 \\
37 A\end{array}$ & $\begin{array}{l}2,96 \pm \\
0,37 \\
\text { B }\end{array}$ \\
\hline $\begin{array}{l}\text { SR } \\
D_{-} \\
F_{-}\end{array}$ & $\begin{array}{l}2,10 \pm 0, \\
22 B\end{array}$ & $\begin{array}{l}117,78 \pm \\
12,58 \mathrm{~B}\end{array}$ & $\begin{array}{l}199,80 \pm \\
21,34 \mathrm{~B}\end{array}$ & $\begin{array}{l}102,41 \pm 4 \\
87 \mathrm{~A}\end{array}$ & $\begin{array}{l}2,91 \pm \\
0,46 \\
\text { B }\end{array}$ \\
\hline
\end{tabular}

Médias com letras iguais na mesma coluna não diferem estatisticamente pelo teste de Tukey ( $p>0,05)$. AR: Árabe; CR: Crioulo; PA: Pantaneiro; QM: Quarto de Milha; SRD: equinos mestiços; MU: Muar. PC: peso corporal; IRCG: índice de relação cernelha e garupa; IDT: índice dáctilo torácico; IC: índice corporal; IM: índice meloscópico; ICF: índice de conformação; ICG1: índice de carga 1; ICG2: índice de carga 2; ICR: índice corporal relativo; ICO: índice de compacidade.

Para pesos corporais, valores superiores a $550 \mathrm{~kg}$ correspondem a cavalos grandes ou considerados hipermétricos; entre 350 e 550 kg, cavalos médios ou eumétricos; e inferiores a $350 \mathrm{~kg}$ correspondem a cavalos pequenos ou elipométricos (Oom e Ferreira, 1987). Assim, foi possível verificar que o Árabe e Quarto de Milha fêmea apresentam perfis hipermétricos, enquanto que os demais grupos genéticos, um perfil eumétrico.

Já o índice dáctilo-torácico, classifica-se como equino em hipermétrico (grande porte) quando o valor é superior a 0,11, eumétrico (médio porte) quando o valor é de 0,105 a 0,108, e por fim hipométrico (pequeno porte) quando o valor é inferior a 0,105 (Cabral et al., 2004). Desta forma, os animais Crioulos machos, Muares e Quarto de Milha foram classificados como hipermétricos, os demais eumétricos. Esse índice relaciona o perímetro do tórax com o da canela, nessas primícias, para que se tenha uma proporção adequada, têm-se a necessidade do desenvolvimento dos membros de acordo com o do tronco (Ribeiro, 1989; Torres e Jardim, 1992).

Através do índice corporal, tem-se informação do equídeo com aptidões mais adequadas para velocidade (longilíneo) quando o valor for superior a 0,90, força (brevilíneos) quando o valor for inferior a 0,85 e intermediária entre força e velocidade (mediolíneos) quando o valor for entre 0,86 e 0,88 . Para este índice, todos os animais apresentaram perfil brevilíneo, com destaque acentuado para Quarto de Milha fêmea. Este resultado concordou com 0 verificado no índice meloscópio, pois animais longelíneos possuem valor superior a 1 , mediolíneo igual a $1 \mathrm{e}$ brevilíneo valor inferior a 1 (Oom e Ferreira, 1987), sendo todos inferior a 1. Portanto, a população dos equídeos estudados possui variações da modelagem corporal, todavia para índices que relatam a habilidade (força, velocidade ou dupla aptidão), os resultados não foram suficientes para categorizar um tipo definido para cada grupo genético, ao ponto que todos se enquadram como brevilíneos, sendo evidenciada apenas variações quanto a maior ou menor quantidade de força. Todavia, isso ocorre principalmente pelo maior porte e/ou estrutura corporal desses animais; e vale ressaltar que em condições Pantaneiras, um animal grande pode possuir uma menor capacidade adaptativa, além de maiores necessidades de manejo principalmente nutricional e isso deve ser levado em consideração pelo produtor no momento da escolha do grupo genético do animal.

Equídeos de sela apresentam índice de conformação igual a 2,11, ao passo que para tração, valores 

superiores a este. Assim, fêmeas Quarto de Milha apresentaram maiores aptidões para capacidade de tracionar pesos mais elevados em relação aos demais grupos. Já os valores de índice de carga 1 e 2, fornecem um indicativo do peso em quilogramas sem esforço exagerado que o animal pode suportar sobre o dorso, trabalhando a trote ou a galope, e trabalhando ao passo. Os animais Quarto de Milha fêmeas em ambos os índices superam os demais grupos, exceto ao Árabe fêmea.

O índice corporal relativo é a relação entre o comprimento do corpo e altura. Quanto mais próximo de 1 for 0 resultado, maior há equilíbrio entre comprimento e altura do corpo. Assim, animais Quarto de Milha e Árabe tendem a serem mais altos do que compridos em comparação aos demais grupos. De acordo com o índice de compacidade 1, equinos com aptidões para tracionarem pesos elevados devem apresentar valores superiores a 3,15; valores próximos a 2,75 indicam equino apto para tração ligeira e próximos a 2,60, sela; considerando o índice de compacidade 2 , valores acima de 9,5 indicam equino apto para tração pesada; entre 8,0 e 9,5, tração leve e 6,0 a7,75, sela. Assim, os animais Àrabes e Quarto de Milha fêmeas apresentaram maior aptidão para cargas mais elevadas em relação aos demais grupos.

Foram verificadas diferenças $(P<0,05)$ entre os grupos genéticos considerando simultaneamente os índices de conformação corporal. Os dois primeiros componentes foram suficientes para explicar $75,23 \%$ de variação entre os animais. O primeiro componente $(59,69 \%)$ foi descrito como: $0,39^{*} \mathrm{P}+0,17^{\star} \mathrm{IRCG}-0,08^{*} \mathrm{IDT}-0,36^{*} \mathrm{IC}$ $+0,04 * \mathrm{IM}+0,40^{*} \mathrm{ICF}+0,40 * \mathrm{ICG} 1+$ $0,40 * I C G 2-0,20 * I C R+0,40 * I C O$. A distribuição dos coeficientes dados aos índices indica que animais mais pesados e brevilíneos, possuem maior capacidade para atividades que exigem força e/ou tração, enquanto que animais longilíneos estão mais aptos a trabalhos com sela.

O segundo componente $(15,54 \%)$ foi descrito como: $0,01^{\star} P+0,53^{\star} I R C G+$ $0,42^{*}$ IDT - 0,13*IC + 0,35*IM - 0,16*ICF - 0,16*ICG1 - 0,16*ICG2 - 0,56*ICR $0,09^{*}$ ICO. Nesse componente, os índices com maiores destaques foram IRCG, IDT e ICR, indicando que dentro dos animais estudados, existem indivíduos hipermétricos e com maiores altura do anterior em relação ao posterior, todavia com modelo corporal menos compacto (mais alto do que compridos). Esta conformação pode não ser interessante, visto que equinos bem proporcionados devem ser tão altos quanto compridos, assim a razão da altura de cernelha com o comprimento do corpo apresenta-se igual a 1 (Oom e Ferreira, 1987).

Nota-se por meio da distância euclidiana no heatmap que três subgrupos são fortemente suportados pela análise (Figura 1). Ficam evidentes as diferenças das fêmeas Árabe e Quarto de Milha dos demais grupos, principalmente por associarem a maiores valores para índices que reportam aptidões a força. Já os demais grupos genéticos sustentam um grupo maior, categorizado por apresentar valores próximos a boa parte dos índices. Outro fato observado foi a distância euclidiana entre os equinos e muares machos mestiços com a raça local, o que pode indicar pouco uso desta na formação desses animais, ou seja, os produtores também têm optado por trabalhar com raças exóticas (Crioulo e Quarto de Milha). 



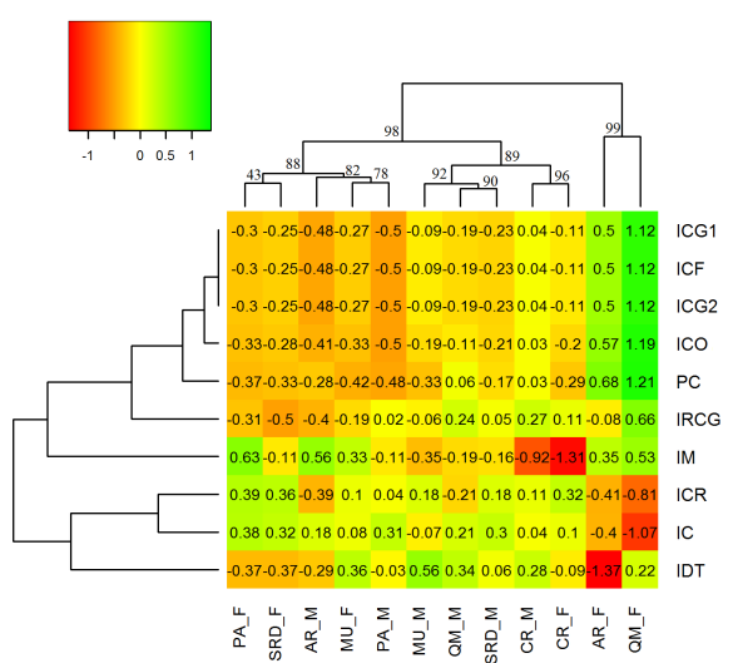

Figura 1. Heatmap dos equídeos criados no Pantanal considerando os índices de conformação corporal.

\section{CONCLUSÃO}

Os índices de conformação corporal apresentam diferenças entre os grupos genéticos de equídeos criados no Pantanal, o que possibilita para o criador, a escolha de um animal com perfil mais adequado a sua necessidade. Pode-se hipotetizar que os produtores têm optado por utilizar raças exóticas na produção de equinos e muares mestiços, visto o menor distanciamento encontrado entre as raças Crioula e Quarto de Milha com esses, e maior com a raça local Pantaneira.

Apesar dos equinos Pantaneiros apresentarem menores aptidões para atividades que exijam força e capacidade de carga em relação ás raças exóticas, não houve diferença de classificação suficiente para mudar o tipo corporal do animal (brevilíneo, mediolíneo e longilíneo), e considerando que esta raça possui perfil eumétrico (médio porte) e já adaptada às condições do Pantanal, os produtores podem optar o seu uso quando se busca alinhar também a rusticidade ao rebanho.

\section{REFERÊNCIAS}

BIAGIOTTI, D.; SARMENTO, J.R.L.; Ó, A.O. et al. Phenotypic characterization of Santa Inês sheep in the State of Piaúi. Revista Brasileira de Saúde e Produção Animal, v.14, n.1, p.29-42, 2013.

CABRAL, G.C.; ALMEIDA, F. Q.; QUIRINO, C.R. et al. Avaliação Morfométrica de Equinos da Raça Mangalarga Marchador: Índices de Conformação e Proporções Corporais. Revista Brasileira de Zootecnia, v.33, n.6, p.1798-1805, 2004.

FRANCI, O.; GIORGETTI, A; GREMOLI, G. et al. Evoluzione delle caractteristiche morphologique nel cavalo avelignese in accrescimento. Zootecnia Nutrizione Animale, v.15, p.373-380, 1989.

HAMMER, Q.; HARPER, D.A.T.; RYAN, P.D. PAST: Palaeontologia Statistics software package for education and data analysis. Palaentologia Eletronica, v.4, 2001.

MCMANUS, C.; FALCÃO, R.A.; SPRITZE, A. et al. Caracterização morfológica de equinos da raça Campeiro. Revista Brasileira de Zootecnia, v.34, n.5, p.1553-1562, 2005.

NICOLLETI, J.L.M.; SCHLEGEL, C.; THOMASSIAN, A. et al. Mensuração do casco de equinos para identificação objetiva de anormalidades de conformação. Veterinária Notícias, v.6, n.1, p.61-68, 2000.

OOM, M.M.; FERREIRA, J.C. Estudo biométrico do cavalo Alter. Revista Portuguesa de Ciências Veterinárias, v.83, n.1, p.101-148, 1987.

PUNDIR, R.K.; SINGH, P.K.; SINGH, K.P. et al. Factor Analysis of Biometric Traits of Kankrej Cows to Explain Body 

Conformation. Asian-Australasian Journal of Animal Sciences, v.24, n. 4, p.449-456, 2011.

R Development Core Team 2008 R: A language and environment for statistical computing.

REZENDE, M.P.G.; SOUZA, J.C. ; CARNEIRO, P.L.S. et al. Morphofunctional Diversity of Equine of Varied Genetic Compositions Raised in the Pantanal Biome of Brazil. Tropical Animal Health and Production, v. 50, p. 1-8, 2018.

REZENDE, M.P.G.; SOUZA, J.C.; MOTA, M.F. et al. Conformação corporal de equinos de diferentes grupos genéticos. Ciência Animal Brasileira, v.17, p.316-326, 2016.

REZENDE, M.P.G.; ABREU, U.G.P.; SOUZA, J.C. et al. Body morphology of purebred and crossbred Quarter horses used in Lasso competitions in Mato Grosso do Sul. Archivos de Zootecnia, v.64, n.246, p.183-186, 2015.

RIBEIRO, D.B. O Cavalo: raças, qualidades e defeitos. 2st ed. São Paulo: Editora Globo. 1989. 318p.

SOUZA, J.C.; REZENDE, M.P.G.; RAMIRES, G.G. et al. Phenotypic traits of equines raised in the Pantanal of Mato Grosso do Sul. Semina: Ciências Agrárias, v.36, n.5, p.3341-3352, 2015.

Statistical analisy System SAS 2000 User's Guide: statistics. Version 8.2. Cary, NC: Sas Institute Inc.

TEIXEIRA NETO, M.R.; CRUZ, J.F.; CARNEIRO, P.L.S. et al. Multivariate analysis of phenotypic diversity in Santa Ines sheep. Revista Brasileira de Saúde e Produção Animal, v,16, n.4, p.784-795, 2015.
TORRES, A.P.; JARDIM, W.R. Criação do cavalo e de outros equinos. 3st ed. São Paulo: Editora Nobel. 1992. 654p.

TORRES, A.P.; JARDIM, W.R. Criação do cavalo e de outros equinos. 3.ed. . São Paulo: Editora Nobel. 1981. 